\title{
Geriatric Anesthesia
}

\author{
Ishrat Yousuf ${ }^{1}$, Pranav Bansal ${ }^{2}$
}

1-DNB Resident, Anesthesiology \& Critical Care, BPS Govt. Medical College, Khanpur Kalan, Sonepat, Haryana, India.

2-HoD, Professor of Anesthesiology \& Critical Care, BPS Govt. Medical College, Khanpur Kalan, Sonepat, Haryana, India.

An 80 y old, geriatric patient has had chronic degenerative disease of the hip joint and has been scheduled for hip replacement surgery. He presents to you for pre-anesthetic checkup. (Please choose the one best option)

Q 1. On cardiovascular examination all of the following are normal physiological findings in a geriatric patient except:-
(A) Increased resting sympathetic nervous system activity
(B) Higher diastolic blood pressure
(C) Left ventricular hypertrophy
(D) Decreased heart rate and cardiac output

Q 2. Which of the following is not an age related change in respiratory mechanics:-
(A) Increased anatomical dead space
(B) Increased closing capacity
(C) Decreased FEV 1
(D) Decreased total lung capacity

Q 3. Which of the following statement is correct regarding structural changes in a geriatric patient:-
(A) Chest wall compliance is not altered
(B) Serum creatinine is a poor indicator of renal function due to decreased muscle mass
(C) An increase occurs in the volume of both grey matter and white matter in the central nervous system
(D) Thermoregulation is not impaired

Q 4. Which of the following is the criteria for inclusion in geriatric age group?
(A) Age $>55 \mathrm{y}$
(B) Age $>60 y$
(C) Age $>65 y$
(D) Age $>70 \mathrm{y}$

Q 5. Delayed recovery of consciousness after anesthesia is common in geriatric age group due to all of the following factors except:-
(A) Increased excretion of drugs like sedatives, hypnotics
(B) Decline in hepatic function
(C) Hypercarbia
(D) Decreased central neuronal activity

Q 6. The term "oldest old" is used for geriatric patients with age:-
(A) $>75 y$
(B) $>80 \mathrm{y}$
(C) $>85 \mathrm{y}$
(D) $>90 \mathrm{y}$

Q 7. Frailty is a clinical syndrome where 3 or more of the following characters are present except:-
(A) Unintentional weight loss $<4 \mathrm{~kg}$ in a y
(B) Unintentional weight loss $>4 \mathrm{~kg}$ in a y
(C) Self-reported exhaustion and weakness
(D) Low physical activity

Q 8. Edmonton Frail Scale includes all of the following except
(A) Cognition
(B) Nutrition
(C) Functional performance
(D) Blood pressure 
Q 9. Which of the following value best corresponds to the progressive decline in cardiac output due to ageing?
(A) $1 \% / \mathrm{y}$ after $30 \mathrm{y}$
(B) $2 \% / y$ after $30 \mathrm{y}$
(C) $3 \% / y$ after $30 \mathrm{y}$
(D) $4 \% / y$ after $30 y$

Q10. All are correct regarding "Get up and Go" test except:-
(A) The person is asked to walk a distance of $3 \mathrm{~m}$.
(B) Duration of $>20 \mathrm{sec}$ implies poor functional performance
(C) It is devised to assess falls risk in elderly
(D) The test is performed without a timed assistance

\section{Explanations:}

A 1(B). The morphologic changes due to aging include decreased myocyte number, thickening of the left ventricular wall, and decreases in both conduction fiber density and the number of sinus node cells.

Functionally, these changes translate to decreased contractility, increased myocardial stiffness and ventricular filling pressures, and decreased $\beta$ adrenergic sensitivity. There is hyperplasia of intima, collagenation of media and accumulation of calcium and phosphate in elastic fibers of vessels resulting in narrowing and an increase in systolic pressure of 10 to $15 \mathrm{mmHg}$ between the central aorta and the periphery, with a slight decrease in diastolic and mean pressures.

A 2(D). Ageing results in increase in the anatomical dead space, decrease tidal volume, increase respiratory rate, decrease vital capacity, increase residual volume, increased closing capacity, decreased $\mathrm{FEV}_{1}$ but no change in total lung capacity.

A 3(B). Elderly patients have decreased muscle mass thus the total serum creatinine level is less. Hence, it is not a reliable factor for renal function as it may underestimate the creatinine levels in renal dysfunction.

A 4(C). A person is said to be in geriatric age when age is $>65 \mathrm{y}$.

A 5(A). Delayed recovery of consciousness after anesthesia is common in geriatric age group due to decreased excretion of drugs like sedatives, hypnotics as a result of age related decrease in GFR, hypoxia, hypercarbia, cerebral thrombosis and associated comorbidities.

A 6(D). A person is classified to be oldest-old when age is $90 \mathrm{y}$ or more.

A 7(A). Frailty is a clinical syndrome where 3 or more of the following characters are present Unintentional weight loss $>4 \mathrm{kgs}$ in a y, self-reported exhaustion and weakness, low physical activity

A 8(D). Edmonton Frail Scale includes 9 criteria i.e. cognition, general health status, functional independence, social support, medication usage, nutrition, mood, continence, functional performance.

A 9(A). The progressive decrease in cardiac output due to ageing is $1 \%$ per $\mathrm{y}$ after $30 \mathrm{y}$.

A 10(D). Get up and go test is devised to assess falls risk in elderly patients. The patient starts in the seated position stands up upon a command walks 3 meters, returns back to the chair and sits down. The time taken for this is recorded and expressed as $0-10 \mathrm{sec}(0$ point $)$ no assistance required, $11-20 \mathrm{sec}$ ( 1 point) and $>20$ $\sec (2$ point $)$ assistance required. 